\title{
Variation of Tasks as a Strategy to Enhance Students' Learning of Algebra
}

\author{
Ng Swee Fong \\ National Institute of Education \\ Nanyang Technological University \\ Singapore
}

\begin{abstract}
Algebra challenges students and teachers alike. Students find algebra abstract. In particular the structural conception of algebra confounds students. Why should the task: (a) simplify $x^{2}+3 x+2$ be any different from; (b) solve $x^{2}+3 x+2=0$ ? Despite their best efforts, teachers find that many students continue to solve task (a) when there is no reason for doing so. Research shows that these two tasks require students to have constructed different meanings of structure and meanings of letters for such algebraic objects. What does it mean to simplify and what does it mean to solve? What meanings do letters have in each of these cases? In this talk, I wish to share how simple yet innovative strategies could be used to help students discern one set of tasks from another. These strategies, underpinned by the theory of variation (Marton \& Tsui, 2004) were tested out by teachers in Singapore. Their work showed that students improved in their performance with various types of algebra tasks. Students' improvements were reflected in terms of their capacity to justify their choices.
\end{abstract}

Key words: Algebra, Sorting Activities, Singapore

\section{Introduction}

It is a fact that algebra is a difficult subject for students to master. For novice learners, their initial encounter with this most mystifying area of mathematics is fraught with confusion. Those conventions acceptable in arithmetic may not necessarily be acceptable in algebra. For example, while it is the norm to write the sum of a whole number and a fraction such as $3+\frac{1}{8}$, as $3 \frac{1}{8}$, this convention cannot be applied to the addition of two letters $a+b$. The sum of this expression is left suspended as a process and its sum can only be determined when the specific values of the letters are provided. Hence $a+b \neq a b$. This example illustrates students' dilemma when to regard an expression as a solution, what Davis (1975) described as a process-product dilemma and that letters are variables, i.e. they represent any numerical value at any given time (Kuchemann, 1981, Usiskin, 1988). It could be said that novice learners of algebra find structural relationships inherent in many algebraic objects most confusing. It is not uncommon to find students erroneously solving quadratic expressions when they were asked to simplify. Erroneous solution in Figure 1 is common amongst novice learners of algebra.

$$
\text { c) } \begin{aligned}
& x^{2}-9 \\
& x^{2}=9 \\
& x= \pm \sqrt{9} \\
& x= \pm 3 \text { or } x=-3
\end{aligned}
$$

Figure 1. Instead of factorizing the quadratic expression, this student chose to solve it instead. 
Also it is not uncommon for students to make the errors listed in Figure 2 when factorising quadratic expressions. Although these students did apply the correct processes yet these processes did not result in the correct equivalent forms of $2(x+1)^{2}$.

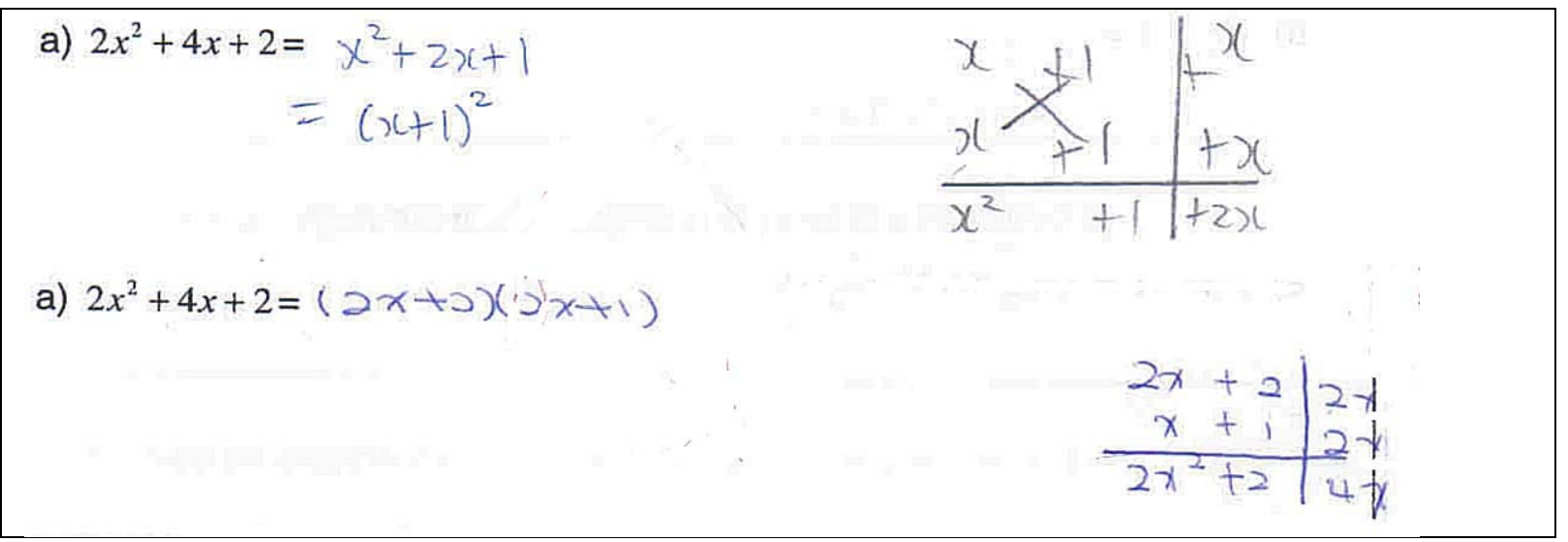

Figure 2. The solution at the top is partly correct but the common factor of 2 is not considered a factor. The solution at the bottom is wrong but the process on the left is correct.

The above examples focused on the symbolic manipulation and transformation aspects of algebra. Although these are not the core of algebra they constitute important skills required to solve problems. Advocates of intensive-computer learning environment which may include using CAS and other handheld technology argue that acquisition of such skills is no longer that crucial as technology could easily perform the processes and thus should not form the core aspect of learning of algebra. Although it may be true that the sophistication of the computers are such that they are able to takeover many of the symbolic manipulative tasks, nevertheless it is equally important for novice learners to master the basics of symbolic manipulation and transformation. I often suspect that such advocates are themselves masters of symbolic manipulation and transformation and when computers are not available, they would be able to apply their knowledge successfully. While it is true that symbolic manipulation and transformation are not the sum total of algebra, such skills are necessary and contribute to the success of problem solving. Also the capacity to carry out the symbolic manipulation and transformation draws from students more knowledge than just the necessary keystrokes. Fey (1984, p. 28) pointed out this difference very clearly.

As procedural operations are increasingly mechanized (by computers), there remains an important task of conceptualization and planning. Problems must still be identified and cast in mathematical form; the proper analyses must be structured and the results of computerassisted calculations must be properly tested and interpreted. To perform this fundamental role, individuals must have a sound understanding of the scope and structure of available mathematical methods.

Although teachers have taught students the necessary symbolic and manipulation skills, students continue to make errors with such basic skills. Some of the errors include but are not limited to the following. 
- Instead of stopping after they have found the factors, many students continued to solve for the unknown values.

- Many students do not know why equations which are contradictions will have no solutions.

- Students are unaware that there are many types of equations.

- Students are confused by the different form of algebraic expressions and hence how to factorise them.

Why do students have this problem? It could be that because teachers may have taught students to be proficient at symbolic manipulation skills and procedures, the many related and similar mathematical objects may cloud students' capacity to differentiate one set of mathematical objects from another. Lack of clarity on the part of students could mean they are unable to differentiate what they do with one set of mathematical object cannot be applied to another almost similar task. Once they have learned new procedures they are unable to inhibit what they have learned to do with one object to another very similar set of objects. Thus such students may benefit from activities which require them to reflect on the similarities and differences that may exist among almost identical objects. Engaging students with such activities may help reduce their impulsiveness and hence improve their performance with such tasks.

In this paper I discuss how different Singapore teachers used sorting activities to help sensitize students to the different expectations of each set of tasks and to improve their performance with these tasks. What are sorting activities? This is will be discussed in the first section of this paper. Why should sorting activity help students improve their knowledge of what they have learned? A theoretical framework is offered to explain why engaging students in sorting activity may contribute to improved performance with basic algebraic skills and procedures. Who conducted these sorting activities? What pedagogical approach did these teachers use? What did students learn from the sorting activities?

\section{What Are Sorting Activities?}

Sorting activities build upon the theory of variation (Marton \& Tsui, 2004). In sorting activities, students are presented different tasks that share certain similar structural properties but are also different in other aspects. To encourage reflection students are asked to use these questions to help them help focus on what are the common aspects of the tasks and what are the differences between the tasks.

What remains the same?

What changes?

What can you do with each object but not with the other?

Because it is not possible to discuss all the different activities, for this paper I will focus only on how to improve students' capacity to differentiate between algebraic equations 
and expressions. In Figure 3, two almost identical mathematical objects are presented for students' consideration: $\mathrm{A}$ is a quadratic expression and $\mathrm{B}$, a quadratic equation. Although $\mathrm{B}$ is an equation, the expression to the left of the equal sign is identical to the expression in $\mathrm{A}$. Although A and B may look alike, there are overt and subtle differences between them. Hence it is the responsibility of the teacher to sensitise students to the variations between these objects. The variations are to address the following learning points:

What are the overt differences? In this case the overt differences could be structural differences: expressions versus equations. Expressions can be factorised but equations can be factorised and solved.

What are the subtle differences? The subtle differences are located within the meanings associated with the letters. In the case of expressions, the resulting equation is an identity which is true for all values of $x$. Unlike expressions, however, equations are only true for specific values of $x$. Hence such equations are known as conditional equations.

$$
\begin{gathered}
\mathrm{A}: \\
\mathrm{x}^{2}+5 \mathrm{x}+6
\end{gathered} \quad \begin{gathered}
\mathrm{B}: \\
\mathrm{x}^{2}+5 \mathrm{x}+6=0
\end{gathered}
$$

Figure 3. Comparing and contrasting two different objects. What remains the same? What changes? Identifying the different processes associated with one but not the other. What can you do with one but not with the other?

Comparing and contrasting two different objects. The two questions: What remains the same? and What changes? focus students' attention on the similarities and differences between two mathematical objects.

What remains the same? This question focused students' attention to the fact that in $B$ the quadratic expression to the left of the equal sign is identical to that of A.

What changes? $\mathrm{B}$ is a quadratic equation but $\mathrm{A}$ is a quadratic expression.

Identifying the different processes associated with one but not the other. What can you do with each object but not with the other? Expression A can be factorised and can be expressed in the form of linear factors. The factorised form of $\mathrm{A}$ is an identity and is true for all values of $x: x^{2}+5 x+6=(x+3)(x+2)$ 
The quadratic expression in B can be factorised and it can be solved by first expressing the quadratic expressions as a product of two linear factors.

$$
\begin{gathered}
x^{2}+5 x+6=0 \\
(x+3)(x+2)=0 \\
x=-3 \text { or } x=-2
\end{gathered}
$$

Hence $\mathrm{B}$ is a conditional equation only true for the two values of $x=-3$ or $x=-2$. How do sorting activities help sensitise students to differences?

\section{A Theoretical Framework}

Research shows that working memory and literacy are two important contributing factors to solving algebraic word problems (Lee, $\mathrm{Ng}, \mathrm{Ng} \& \mathrm{Lim}, 2004$ ) and mathematical problem solving in general (Bull \& Scerif, 2001; Gathercole, et al. 2008). Working memory can be defined as an individual's mental workspace where relevant information is held and to be acted upon. Baddeley and Hitch's (1974) tri-partite working memory model comprising the three components, central executive, phonological loop and visual-spatial sketchpad is used in this discussion. Miyake et al. (2000) building upon work related to working memory (Baddeley, 1996; Baddeley \& Hitch, 1974) fractionated the central executive into three interrelated executive components: Shifting, updating and inhibition. Shifting refers to the capacity to alternate between multiple tasks, operations or mental representations. Updating refers to the capacity to evaluate information and appropriately edit it with more relevant information. The capacity to resist automatic but inappropriate responses describes the last of the three executive functions: inhibition. Literacy refers to students' capacity to decode information presented to them orally.

When confronted with algebraic objects with similar properties, there could be a working memory overload and novice learners of algebra may be unable to shift, update and inhibit their responses to these items. For example at the end of the introductory course to beginning algebra, students would have learnt how to factorise and solve a variety of equations. Because they have so much related information within them, when asked to factorise an expression, they may be unable to shift their attention between different representations and the related operations. They may have difficulty updating the requirement of the task and evaluate what is required of them. Finally they are unable to resist inappropriate responses to the selected task. Hence instead of stopping after they have found the factors to related expressions, these students continue to solve the expression although that was not the demand of the task.

Using sorting activities with students who have completed the introductory algebra course may help sensitise them to the different demands of related yet different algebraic objects, e.g. factorizing versus solving. With sorting activities, two like tasks are presented 
for students to compare and contrast. Guided by these questions: How are the two tasks alike? How are they different? What can you do with one but not the other? Students are asked to compare and contrast these two tasks. Engaging students in such tasks encourages them to reflect on the different demands of each task. When asked to compare and contrast two tasks, students need to shift their attention between the representations of the two tasks, update with the information that equations are to be solved and inhibit the tendency to want to solve expressions. Rather factors are to be found for expressions instead. When they practise with many different similar mathematical objects, they rehearse the mathematical concepts and procedures. With repetition they commit these ideas to long term memory. By repeating such activities, such habits of working become part of the habits of mind and become part of their mathematical disposition. By rehearsing the language used to discuss these mathematical objects and the related processes students' capacity to use mathematical language is enhanced.

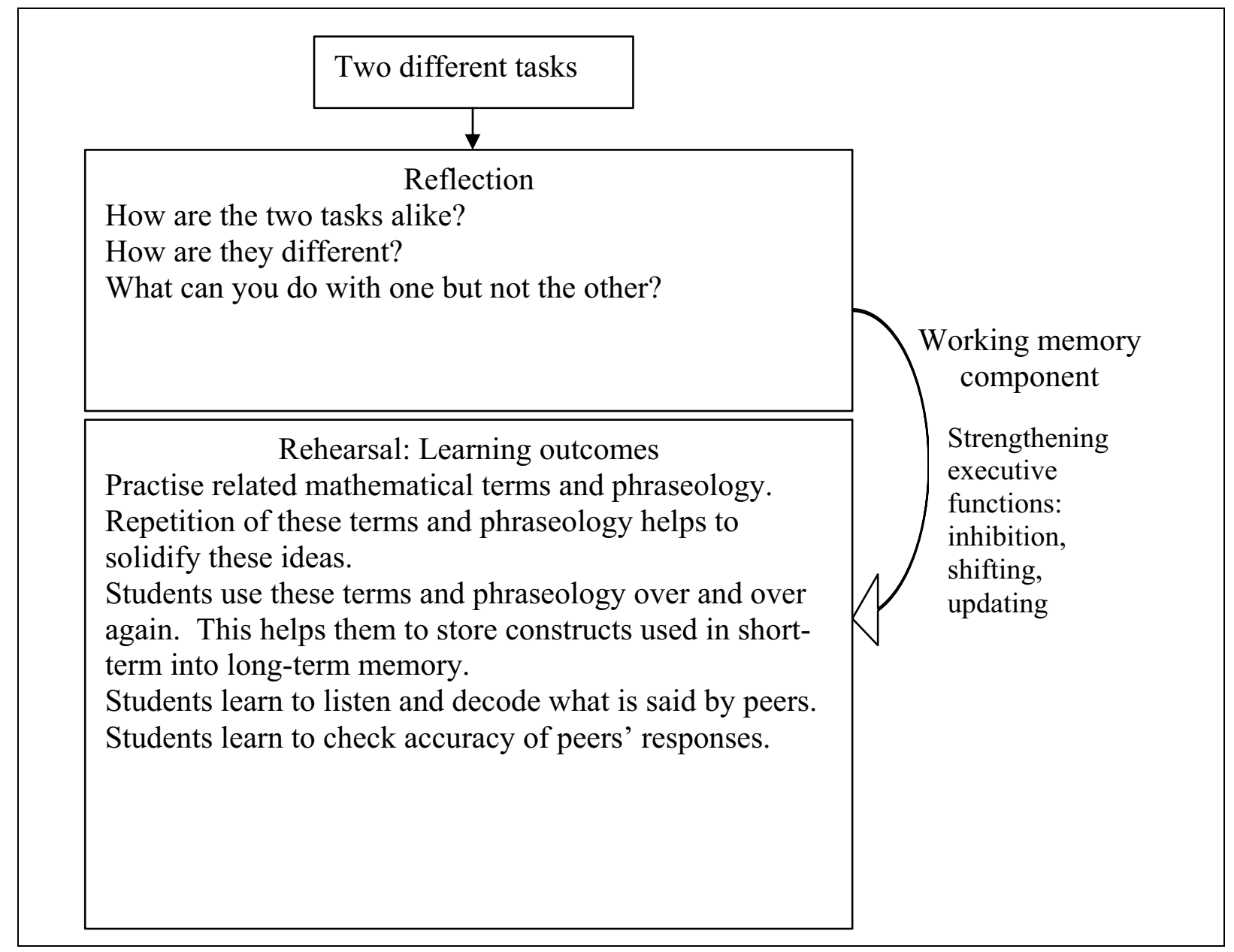

Figure 4. Framework delineating the various processes underpinning sorting activities.

Sorting activities can be conducted with students working on their own or in groups of two. It is best to get students to work in pairs first. The need to justify their answers to their significant other encourages students to speak aloud their thoughts. Why would they 
categorise a certain task as an expression and another an equation? If one member of a pair is unable to start, the significant other could serve as a model of how to articulate their thoughts. This social cultural mode of learning (Vygotski1, 1978) means that the other member of the pair learns from the significant other. The public talk could then become private speech when students are asked to work independently. Unless there is a monitoring system which ensures everyone is actively engaged in the task, it is not advisable to work with too big a group.

In summary the objective of sorting activities is to improve novice students of algebra performance with basic skills by: (i) encouraging students to be more reflective; (ii) strengthening the executive components of the central executive of the working memory; (iii) providing students the opportunities to engage in mathematical talk and the act of listening to mathematical talk, (Mason, Burton \& Stacey), and finally and most important of all; (iv) cultivating a mathematical habit of mind in problem solving. Although the examples cited in this paper are related to letter symbolic manipulation and transformation, sorting activities have found be to very effective in other areas of mathematics. These include sensitizing students to presenting better solutions to the same problem, helping students to avoid common mistakes in solving problems, etc. The diagram in Figure 4 sums up the framework. The flowchart for the sorting task can be found in Appendix B.

\section{Testing the efficacy of sorting activities}

Aliza Main (2006) was the first teacher to use sorting activity as part of her master's dissertation where students were asked to sort and categorise algebraic expressions of varying complexity according to their level of difficulties and the procedures needed to factorise these expressions. The students who participated in this activity explained that the sorting activity sensitized them to the nature of algebraic expressions (linear versus quadratic expressions) and the various procedures needed to factorise them (factorization by common factors involving two terms, factorization by common factors involving three terms, factorization by grouping, factorization of trinomials in the form of $a x^{2}+b x+c$, and factorization of binomials in the form of difference of two squares). Inspired by Main's work, a group of teachers conducted these sorting activities. These teachers were enrolled in the Algebra and the Teaching of Algebra module which was part of the Master of Education Programme offered by the Mathematics and Mathematics Education (MME) academic group of National Institute of Education, Nanyang Technological University of which I was the tutor. As part of the assessment, they were required to demonstrate how they could improve performance in areas which their students consistently found difficult. Although many teachers tried the sorting activity, only the work of Amutha (2010), Chua (2010) and Teo (2010) are included here and because of space only Chua's (2010) work will be cited. The post-test instrument is presented in the appendix. 


\section{Method}

Each teacher identified a problematic area and then designed sorting activities with which they could engage their students. The sorting activity could be based on those that others have done or they could construct sorting tasks that addressed their specific needs. First teachers constructed an instrument to evaluate the knowledge held by their students. An intervention in the form of the sorting activity was conducted. Teachers showed students how to do the sorting activity. Students then worked in groups of 2 to 4 on sets of sorting tasks. After conducting the intervention activity a post-test was carried out to evaluate the efficacy of the sorting task. Also students were asked to provide feedback on what they thought of the sorting activity. The flowchart in Figure 5 provides the design of the study.

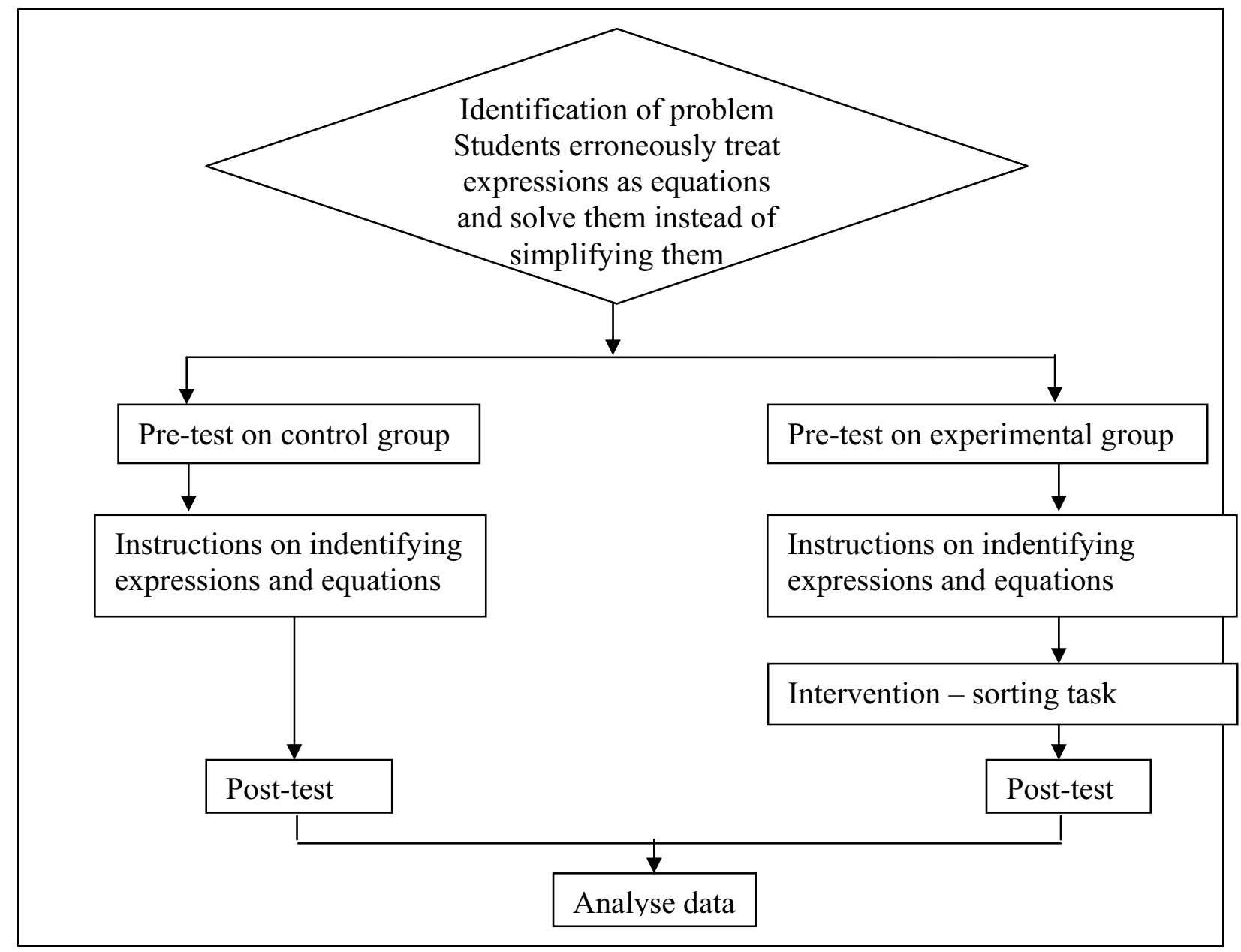

Figure 5. The flowchart provides the design underpinning the intervention studies.

Teachers compared teaching methods. With the control group, teachers used conventional teaching method to review the lessons on factorization and solving of equations while the sorting activity was tried out with the experimental group. Teachers presented the sorting activities to encourage students to reflect upon what differentiates different 
mathematical objects and hence what they can do with one object but not with another. Preand post-tests were carried out to evaluate the efficacy of the teaching methods. Student of similar ability were randomly assigned to each class.

\section{Instrument}

The instrument in Appendix A was the post-test given to the students after the intervention. The first section the instrument required students to work with numerical expressions and equations. The second section focused on algebraic examples.

\section{Findings}

It was expected that students who received the conventional teaching and those who were engaged in the sorting activity benefitted from the teaching. But students who were engaged in the sorting task could state precisely what they could do with one set of objects but not with the other. For example, students were able to state that they could factorise expressions but not solve them. For equations they could factorise as well as solve the equations. Students found the hands-on activity interactive as they could discuss the mathematics involved in carrying out the mathematics task. They learn and remember the mathematics built into the sorting activity better than if they were to listen to the teacher. They enjoyed the learning process.

\section{Conclusions}

In this paper, sorting activities are presented as a means to help improve performance of novice learners with basic algebraic activities. The preliminary findings reported in the four Singapore studies show that sorting activities has the potential to help learners improve their central executive functions as well as their literacy skills, and hence their learning of basic algebraic manipulations. More rigorous research is needed to verify these findings. Nevertheless sorting activities are a useful means for teachers to use as a review tool to encourage students to reflect upon what they have learnt at the end of a section.

\section{References}

Amutha d/o Annathurai (2010). Factorization of algebraic expressions: Identifying the different categories of factorisation improve students' ability in algebraic factorization. Term paper submitted for the capstone module Critical Inquiry MMM800.

Baddeley, A. D. (1996). Exploring the central executive. Quarterly Journal of Experimental Psychology, 49A, 5-28.

Baddeley, A. D., \& Hitch, G. (1974). Working memory. In G. H. Bower (Ed.), The psychology of learning and motivation: Advances in research and theory (Vol. 8, pp. 4789). New York: Academic Press. 
Bull, R., \& Scerif, G. (2001). Executive Functioning as a Predictor of Children's Mathematics Ability: Inhibition, Switching, and Working Memory. Developmental Neuropsychology, 19(3), $273-293$.

Chua, J. (2010). Do engaging students in sorting tasks improve students' capacity to differentiate expressions from equations? Yes and No. Term paper submitted for the capstone module Critical Inquiry MMM800.

Davis, R. B. (1975). Cognitive processes involved in solving simple algebraic equations. Journal of Children's Mathematical Behavior, 1(3), 7 - 35.

Fey, J. T. (Ed.). (1984). Computing and mathematics: The impact on secondary school curricula. College Park: The University of Maryland.

Gathercole, S. E., Alloway, T. P., Kirkwood, H. J., Elliott, J. G., Holmes, J., \& Hilton, K. A. (2008). Attentional and executive function behaviours in children with poor working memory. Learning and Individual Differences, 18(2), 214-223.

Kuchemann, D (1981), ‘Algebra'. In Hart, K. M. (Ed), Children's Understanding of Mathematics: 11-16. London: John Murray; Athenaeum Press Ltd.

Lee, K., Ng, E. L., \& Ng, S. F. (2009). The contributions of working memory and executive functioning to problem representation and solution generation in algebraic word problems. Journal of Educational Psychology, 101(2), 373-387.

Main, A. (2006). Factorization of algebraic expressions: Perceptions and misconceptions held by Secondary 3 Express and Normal Academic students. A dissertation submitted in part fulfilment for the degree of Master of Education, National Institute of Education, Nanyang Technological University. Singapore.

Marton, F., \& Tsui, A. B. M. (2004). Classroom discourse and the space of learning. Mahwah. N.J.: Lawrence Erlbaum.

Mason, J. Burton, L. \& Stacey, K. (1985). Thinking mathematically. Wokingham: UK: Addison-Wesley Pub. Co.

Miyake, A., Friedman, N. P., Emerson, M. J., Witzki, A. H., Howerter, A., \& Wager, T. D. (2000). The unity and diversity of executive functions and their contributions to complex "frontal lobe" tasks: A latent variable analysis. Cognitive Psychology, 41, 49-100.

Teo, L. S. (2010). Students' achievement and difficulties faced in the factorisation of algebraic expressions. Term paper submitted for the capstone module Critical Inquiry MMM800.

Usiskin, Z. (1988). Conceptions of School Algebra and Uses of Variables. In A. F. Coxford \& A. P. Shulbert (Eds.) The Ideas of Algebra, K-12. 1988 Yearbook. Reston, Virginia: National Council of Teachers of Mathematics.

Vygotski1, L. S. (1978). Mind in society: The development of higher psychological processes. Cambridge, Mass.: Harvard University Press. 
Appendix A

\begin{tabular}{|c|c|c|c|c|c|c|}
\hline For each of the following put a & \multirow{2}{*}{\multicolumn{2}{|c|}{ Expression }} & \multicolumn{4}{|c|}{ Equation } \\
\hline tick in the relevant-correct box. & & & \multirow{2}{*}{\multicolumn{2}{|c|}{ Identity }} & \multicolumn{2}{|c|}{ Contradictions } \\
\hline \multicolumn{5}{|l|}{$7=3+4$} & & \\
\hline \multicolumn{7}{|l|}{ 2) $5+4-3$} \\
\hline \multicolumn{7}{|l|}{ 3) $3 \times 2+4$} \\
\hline \multicolumn{7}{|l|}{ 4) $3=3$} \\
\hline \multicolumn{7}{|l|}{ 5) $2 \times 5=5 \times 2$} \\
\hline \multicolumn{7}{|l|}{ 6) $18 \div 3=18 \times \frac{1}{3}$} \\
\hline \multicolumn{7}{|l|}{ 7) $5=5+1$} \\
\hline \multicolumn{7}{|l|}{ 8) $9+3=12+5$} \\
\hline \multicolumn{7}{|l|}{ 9) $12 \div 2=4+2$} \\
\hline \multicolumn{7}{|l|}{ 10) $3 \times 5=30 \div 2$} \\
\hline \multicolumn{7}{|l|}{ 11) $\frac{2}{3}-\frac{1}{2}$} \\
\hline \multicolumn{7}{|l|}{ 12) $\frac{1}{5}+\frac{1}{3}=\frac{8}{15}$} \\
\hline \multicolumn{7}{|l|}{$4(5+10)=4 \times 5+4 \times 10$} \\
\hline & \multicolumn{2}{|c|}{ Expression } & \multicolumn{4}{|c|}{ Equation } \\
\hline & $\begin{array}{l}\text { Like } \\
\text { terms }\end{array}$ & $\begin{array}{l}\text { Unlike } \\
\text { terms }\end{array}$ & Identity & & $\begin{array}{l}\text { ional } \\
\text { tion }\end{array}$ & Contradictions \\
\hline \multicolumn{7}{|l|}{ 1) $3 a+3 a$} \\
\hline \multicolumn{7}{|l|}{ 2) $3 a+2 a$} \\
\hline \multicolumn{7}{|l|}{ 3) $3 a-2 a$} \\
\hline \multicolumn{7}{|l|}{ 4) $3 a+2 b$} \\
\hline \multicolumn{7}{|l|}{ 5) $3 a-2 b$} \\
\hline \multicolumn{7}{|l|}{ 6) $2(a+b)$} \\
\hline \multicolumn{7}{|l|}{ 7) $4 a(a+b)=0$} \\
\hline 8) $5 t^{2}=t$ & & & & & & \\
\hline 9) $3 p(p+2)=3 p^{2}+6 p$ & & & & & & \\
\hline 10) $x=x+1$ & & & & & & \\
\hline 11) $(x+2)(x+1)=x^{2}+3 x+2$ & & & & & & \\
\hline 12) $y \times 0=y$ & & & & & & \\
\hline 13) $\frac{y-1}{2}=\frac{2}{y-1}$ & & & & & & \\
\hline 14) $\frac{5 x^{2}-20}{10 x^{2}+10 x-20}$ & & & & & & \\
\hline 15) $3(x-5)-1=7-(1-x)$ & & & & & & \\
\hline Can you solve the following? & No & Yes & & es, & your 1 & orking \\
\hline 1) $3(x+y)$ & & & & & & \\
\hline 2) $3 x^{2}=x$ & & & & & & \\
\hline 3) $2 a(a+2)=2 a^{2}+4 a$ & & & & & & \\
\hline 4) $4 a=0$ & & & & & & \\
\hline 5) $(x+2)(x+1)$ & & & & & & \\
\hline 6) $x+1=x$ & & & & & & \\
\hline 7) $x+3 x+2=0$ & & & & & & \\
\hline
\end{tabular}




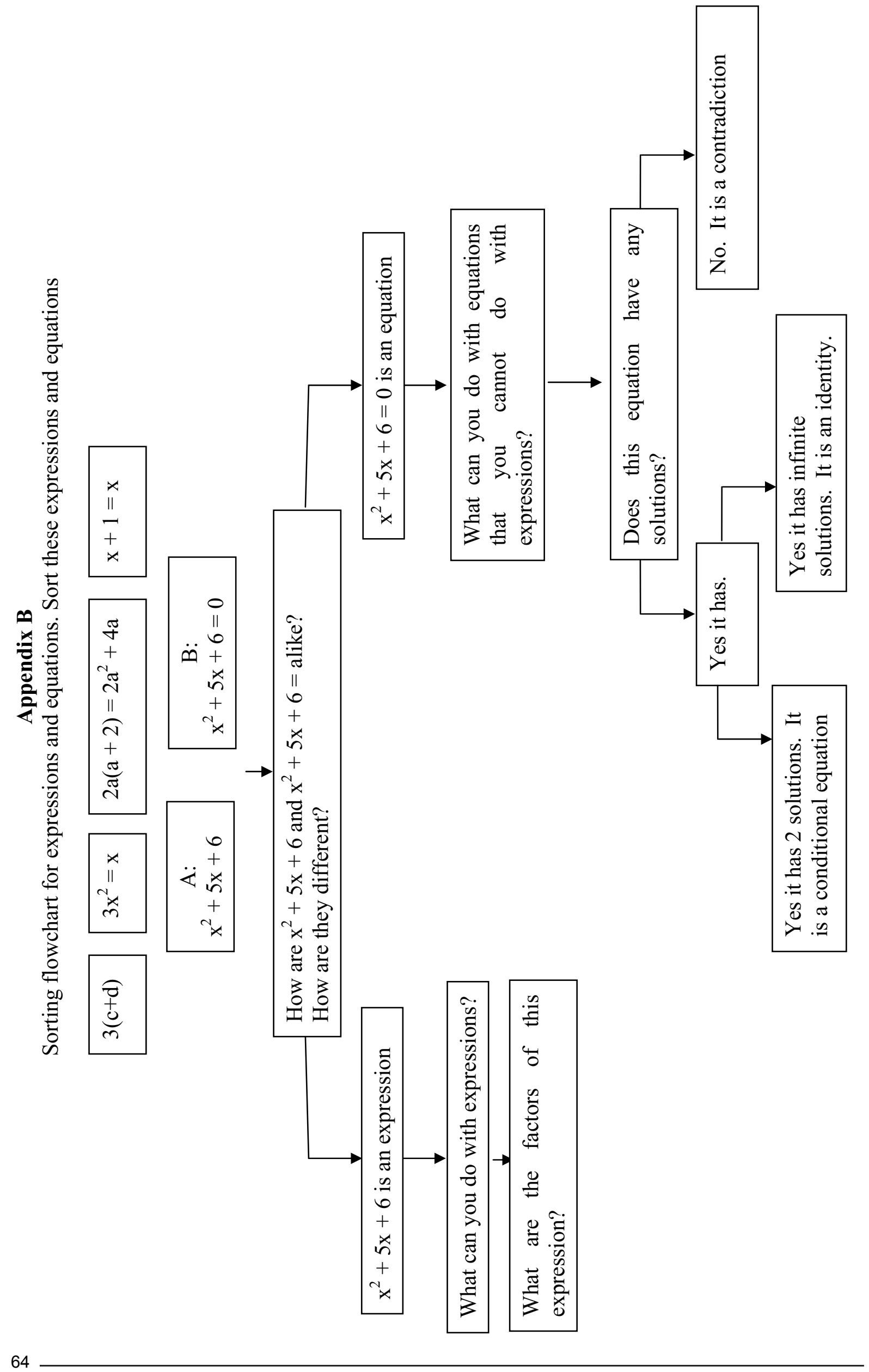

